\title{
APLICACIÓN DE UN MODELO DE EVALUACIÓN A LAS REVISTAS CIENTÍFICAS ESPAÑOLAS DE ECONOMÍA: UNA APROXIMACIÓN METODOLÓGICA
}

\author{
Elea Giménez Toledo*, Adelaida Román Román*, \\ José María Sánchez Nistal*
}

Resumen: Se describe un modelo de evaluación de revistas cientificas, basado en el análisis de parámetros múltiples: cumplimiento de la periodicidad, pervivencia, normalización, consejos de redacción, sistema de evaluación de originales, apertura institucional, difusión internacional y valoración de los pares. Se comentan los aspectos metodologicos más destacables, aś como los resultados globales obtenidos al aplicar este modelo a una gran muestra de las revistas españolas de Economía.

Palabras clave: revistas cientificas, economía, evaluación cientifica, indicadores de calidad, modelos de evaluación de revistas.

Abstract: This paper describe a scientific journal evaluation system based on the analysis of multiple parameters: frequency of publication, historical prestige, standardisation, editorial board, papers evaluation system, origin of contributions, international diffusion and peers evaluation. The most prominent methodological aspects are commented, as well as the results obtained when applying this model to a broad sample of Spanish journals on Economics.

Key words: scientific journals, economics, research evaluation, indicators of quality, journal evaluation models.

\section{Introducción}

El interés que viene despertando desde hace cuatro décadas la evaluación de revistas científicas está justificado por diversas razones. La multiplicidad de títulos de revistas para un mismo campo temático, la escasez de cobertura en otros, las tiradas limitadas que suelen hacerse de muchos de los títulos, o la escasez de medios, en particular de ayudas para las publicaciones científicas, dibujan un escenario empobrecido, no consolidado y con multitud de problemas para subsistir, no sólo en nuestro país.

Las conclusiones que pueden obtenerse de los procesos de evaluación aportan información detallada sobre la situación real de cada publicación, sobre su calidad, su difusión, etc., lo que facilita la toma de decisiones y el establecimiento de medidas para mejorar la situación, así como para orientar las ayudas o subvenciones de la manera más eficaz posible.

Por todos estos motivos, diferentes organismos de distintos países han ensayado algunos modelos, buscando la manera más idónea de evaluar la calidad de las revistas y de actuar como impulsores de la mejora de esa calidad. Todos estos modelos están basados en múltiples indicadores que pretenden eliminar la subjetividad que conlleva cualquier proceso de evaluación.

* CINDOC-CSIC. Correo-e: adelaida@ cindoc.csic.es.

Recibido: 22-3-99. 
En España, el Centro de Información y Documentación Científica (CINDOC) del Consejo Superior de Investigaciones Cientificas (CSIC) ha llevado a cabo algunas experiencias de evaluación de revistas cientificas. En este trabajo se pretende exponer los resultados obtenidos en el estudio valorativo de las revistas científicas españolas de Economía, proyecto que obtuvo financiación por parte de la Dirección General de Enseñanza Superior en el verano de 1997.

\section{Algunos precedentes en la evaluacion de revistas cientificas}

A continuación se citan algunos modelos y métodos de evaluación de revistas científicas. La variedad de posibilidades descritas muestra el enorme interés que el tema despierta. Se pretende describir algunos de los modelos más conocidos y que más se han tenido en cuenta a la hora de establecer los criterios de calidad elegidos para el estudio evaluativo de las revistas españolas de Economía. No se trata pues de una revisión sobre el tema, sino tan sólo de establecer, mediante unas pinceladas descriptivas muy someras, el entorno metodológico más próximo al trabajo que se presenta.

Los países latinoamericanos han sido muy activos en la última década a la hora de desarrollar modelos de evaluación de revistas científicas y técnicas debido a que les afectan muchos de los problemas citados anteriormente.

Krzyzanowski y otros autores (1) remiten a algunos de los métodos empleados en América Latina. Así, hacen referencia al modelo diseñado por el grupo de trabajo para la selección de revistas técnicas latinoamericanas de UNESCO en 1964, en el que se trabajaba con variables como la presentación de las publicaciones, la duración, la regularidad, la periodicidad, la aceptación de colaboradores de otras instituciones, el nivel de especialización y la inclusión en índices, entre otras. Este mismo modelo sirvió de base, en 1968, para llevar a cabo una evaluación de las publicaciones médicas venezolanas, y más tarde (1982) fue adaptado como sistema de evaluación de publicaciones brasileñas técnicas y científicas, asignando puntuaciones a las distintas variables. La misma autora cita un proyecto más amplio, desarrollado por el Centro Latinoamericano de Información sobre Ciencias de la Salud (BIREME), en el que se analizaron las publicaciones latinoamericanas clasificadas en los índices Medline y Lilacs; el objetivo era volver a evaluar las revistas que ya estaban incluidas en esos índices y, por otra parte, evaluar revistas que no estaban incluidas pero que podrían incorporarse.

Según los datos de los que disponemos, quizás sea Brasil el país más productivo en cuanto a estudios de evaluación de revistas se refiere. Así, en 1983 se inicia el Programa Sectorial de Publicaciones en Ciencia y Tecnología, fruto de la colaboración entre el Centro Nacional de Desarrollo Científico y Tecnológico (CNPq) y la Financiadora de Estudios y Proyectos (FINEP). A través de este estudio se pretendía evaluar las revistas financiadas por esta última institución. En 1994, Moriconi Valerio (2) publícó una obra en la que se planteaban multitud de criterios que podrían tenerse en cuenta para evaluar revistas científicas. Esta obra tiene como contexto el Programa Sectorial citado anteriormente.

Más tarde, en 1988 y en 1991 se realizaron dos estudios interesados fundamentalmente por la evaluación del contenido («mérito») de las publicaciones. Para ello se recurría a la opinión de los pares, que daban las pautas para definir un núcleo de revistas científicas nacionales al que irían destinadas las ayudas económicas de FAPESP (Fundación de Amparo a la Investigación del Estado de São Paulo). 
Para cerrar el bloque dedicado a Brasil, ha de mencionarse el modelo presentado en el II Taller sobre publicaciones científicas en América Latina (Guadalajara, México, 1997) dirigido nuevamente a evaluar las publicaciones científicas y técnicas nacionales, y caracterizado por el empleo de numerosos indicadores y por la asignación de puntuaciones a cada variable estudiada.

En este mismo taller también se dio a conocer el modelo elaborado por el grupo de Cienciometría de Colciencias (Instituto Colombiano para el Desarrollo de la Ciencia y la Tencologia «Francisco José de Caldas») (3). Este modelo trabaja también con múltiples variables reunidas en torno a cuatro grandes apartados: calidad editorial, normalización, visibilidad nacional y visibilidad internacional. Para cada uno de ellos se propone una formula matemática, que opera con los valores de las variables obtenidos y que sirve para evaluar globalmente las revistas desde esos cuatro ángulos.

Por otra parte, también se tiene noticia de los trabajos llevados a cabo en este sentido en Venezuela, así como en México para la elaboración de un índice de revistas cientificas de excelencia.

Finalmente es necesario hacer mención del proyecto LATINDEX: f́ndice Latinoamericano de Publicaciones Científicas. El objetivo de este proyecto es crear una base de datos para difundir los trabajos que se publican en las revistas latinoamericanas y que sirva además para realizar trabajos bibliométricos. Para alcanzar este objetivo se han definido dos etapas previas que buscan respectivamente la elaboración de un inventario de todas las publicaciones científicas producidas en todos los países de América Latina y la elaboración de un catálogo que recoja tan sólo aquellas publicaciones del anterior inventario que cumplan con unos mínimos requisitos de calidad. Los trabajos publicados en las revistas de este catálogo son los que formarían parte de la base de datos.

Hasta el momento, algunos de los criterios de calidad que se han propuesto son: cumplimiento de las normas ISO, cumplimiento de la periodicidad, mecanismos de selección de originales mediante revisores y difusión de las revistas en función de su aparición o no en servicios de indización y resumen. Este mismo año se celebrará un encuentro en el que se decidirán los criterios que finalmente se aplicarán teniendo en cuenta el objetivo pretendido.

A finales de los años setenta empiezan a realizarse en España algunos estudios sobre la calidad de las revistas científicas y técnicas. Analizando un poco la evolución que han experimentado estos estudios, destaca el aumento de los parámetros utilizados para la evaluación de revistas. Cabe señalar una dedicación mucho más acentuada hacia todas las disciplinas científico-tecnológicas, con trabajos orientados a estudiar de una manera especial los aspectos ligados a la visibilidad y difusión internacional de las revistas (4) (5) y la escasez de estudios de valoración de revistas de Ciencias Sociales y Humanidades.

Tal y como indica Delgado López-Cozar en su revisión bibliográfica (6), los estudios de evaluación de revistas realizados en España han trabajado fundamentalmente con tres tipos de criterios: criterios formales, criterios de contenido científico y criterios de difusión.

Para matizar un poco esta información, es conveniente señalar que los estudios de evaluación más recientes para las revistas españolas de Ciencia y Tecnología han trabajado con distintos indicadores: número de revistas por campo científico, tipo de editores, periodicidad y pervivencia, adecuación a las normas internacionales de presentación de publicaciones periódicas, producción de las revistas, presencia en bases de 
datos internacionales y otros servicios secundarios de información, contribuciones de autores extranjeros, coautorías internacionales, análisis de citas y estudio de los hábitos de publicación de los investigadores.

Esta misma metodología con la combinación de distintos indicadores ha sido la base para el estudio de las revistas españolas en algunas disciplinas de Ciencias Sociales y Humanidades. Más concretamente, en 1995 se abordo el estudio de la calidad de las revistas de Sociología, Prehistoria e Historia Antigua y Arqueología (7) (8). El método que se utilizó en aquella ocasión ha sido notablemente modificado en la valoración de las revistas españolas de Economía. En aquella ocasión no se aplicaron criterios de calidad formal que ahora se incorporan y se abordo un estudio de citas, en el que se puso de manifiesto su gran interés y su dificultad metodológica, dadas las prácticas de citación de los investigadores sociales y de Humanidades. Esta experiencia y el conocimiento cercano de otras, determinaron un replanteamiento metodológico. Los indicadores que han formado parte del modelo de evaluación aplicado a las revistas de Economía se describen detalladamente en el apartado 3 de este trabajo.

En el ámbito europeo, llama la atención la escasez de estudios de evaluación de revistas científicas 0 , al menos, su poca visibilidad. No obstante, hay que destacar los trabajos en el área de la Contabilidad, de Jones, Brinn y Pendlebury (9) de la Escuela de Negocios de Cardiff. Proponen el análisis de citas y los estudios de valoración de los pares como pilares fundamentales en la evaluación de revistas. Ambos análisis presentan ventajas e inconvenientes, pero estos autores se inclinan más hacia la aportación de los pares porque consideran que es más completa y global que el análisis de citas. Proponen que se consulte también a investigadores extranjeros y no sólo a investigadores del propio país, con el objeto de afinar más en las conclusiones y evitar así posibles sesgos. Frente a estas ventajas, está la irremediable subjetividad que se desprende de los juicios personales de los investigadores. En este sentido, argumentan que los análisis de citas son más objetivos pero no están exentos de limitaciones. Según afirman en su artículo, las citas no siempre reflejan fielmente el consumo de información científica por parte de los investigadores para realizar sus trabajos, ni están claras del todo, por otra parte, las causas por las que determinadas revistas quedan excluidas del circuito de citas y, por tanto, de las bases de datos del ISI.

Especialmente interesante es el modelo de evaluación de revistas propuesto por el Consejo Nacional de Investigación de Canadá (10). Se caracteriza porque contempla diversos aspectos relacionados con el contenido científico de la revista, con la aceptación de la misma por parte de la comunidad científica y con la calidad de la edición. Tiene en cuenta, entre otras cosas, la opinión de los especialistas en la materia y los análisis de citas. No valora el cumplimiento o incumplimiento de las normas internacionales que afectan a las publicaciones periódicas.

Resulta inevitable detenerse en los criterios de evaluación de revistas empleados por el Institute for Scientific Information (ISI) (11) para seleccionar las publicaciones que forman parte de sus bases de datos. El objetivo de la evaluación que llevan a cabo es la elaboración de un producto documental de especial interés porque recoge las citas de los documentos que analiza. Sin embargo, traspasando este objetivo inicial, las bases de datos del ISI han servido como referencia fundamental para la evaluación de la actividad científica de los investigadores. No se puede considerar, por tanto, que los criterios empleados conformen un modelo como tal, comparable con el resto de los que se están citando. 
El ISI aplica sus criterios de evaluación a revistas de todo el mundo y de todas las disciplinas científicas. Esto implica un cuidadoso proceso de selección que procede de la estricta aplicación de los siguientes parámetros:

- Normalización: para que una revista sea seleccionada e incluida en las bases de datos del ISI es necesario que cumpla la periodicidad, que tenga un título suficientemente representativo del contenido, que incluya títulos y resúmenes de los artículos significativos, que ofrezca información bibliográfica completa para todas las citas y que presente la afiliación institucional completa del autor. Por otra parte, es esencial que los títulos de los artículos, los resúmenes y las palabras clave estén en inglés, además del idioma original. Por último, se valora positivamente el hecho de que la revista utilice como filtro de calidad un sistema de evaluación por pares.

- Análisis de citas: se utiliza de forma distinta según se trate de revistas ya incluidas, para las cuales se observa el índice de citación, el factor de impacto y el índice de inmediatez, o de revistas por incluir, para las que se analiza el impacto de las publicaciones de los autores y de los miembros del consejo de redacción de esa revista.

- Contenido editorial: se trata de conseguir una base de datos equilibrada en la que estén cubiertas todas las materias. Por este motivo, y con el fin de no duplicar información, antes de incluir una revista nueva se analiza si la materia que trata está o no suficientemente cubierta por la base de datos. Al menos, según Testa (11), una revista muy buena de una materia ya suficientemente cubierta por el ISI tendría dificultades para ser aceptada.

- Internacionalidad: también se pretenden recoger revistas de todos los ámbitos geográficos. Según Testa (11) las revistas regionales para ser incluidas no se comparan con las de su especialidad sino con las de su misma área geográfica.

Mientras que los dos primeros aspectos son comunes a varios modelos de evaluación de revistas, el contenido editorial y la internacionalidad no lo son y tienen consecuencias en la desigual cobertura temática y geográfica de las bases de datos del ISI, a pesar de que este instituto selecciona revistas de todos los campos científicos y procedentes de muchos países distintos.

\section{Las revistas cientificas españolas de Economía: metodología para su evaluación}

\subsection{Revistas sometidas a evaluación}

Como ya se ha indicado anteriormente, el estudio que aquí se presenta se circunscribe al área de Economía. Se han tomado las revistas recogidas sistemáticamente por la base de datos ISOC, que recoge 103 títulos. El área de Economía del CINDOC seleccionó aquéllos de mayor contenido científico y menos coyunturales para su evaluación: la lista propuesta inicialmente está formada por 48 revistas. Se ha hecho el estudio con los números editados en 1995 y 1996 y manejando directamente los ejemplares. 


\subsection{Criterios de calidad empleados para la valoración de las revistas}

El estudio de las revistas se ha hecho analizando el cumplimiento de diferentes parámetros de calidad que pueden agruparse en las siguientes categorías:

1. Parámetros que miden la calidad formal: periodicidad, pervivencia, normalización.

2. Parámetros que miden la calidad de contenidos de manera indirecta: composición de los Consejos de Redacción, sistema de selección de originales, apertura exterior (origen institucional de las contribuciones aceptadas para publicar), difusión internacional de la revista.

3. Juicio de los «pares» sobre la calidad de contenido de las revistas.

A continuación se explican los criterios seguidos para aplicar los diferentes parámetros citados:

Periodicidad. Para evaluar el grado de cumplimiento de la periodicidad de una revista se han tenido en cuenta dos criterios complementarios: a) si se han editado en el año un número de fascículos acorde con la periodicidad anunciada en la revista; b) la fecha de llegada de los fascículos a un centro de información. Esta fecha de recepción se ha tomado como referencia para estimar el cumplimiento de la periodicidad si:

- Las revistas mensuales o bimensuales llegaron a la biblioteca en un plazo no superior a un mes después de su publicación.

- Las revistas trimestrales y cuatrimestrales llegaron a la biblioteca en un plazo no superior a dos meses después de su publicación.

- Las revistas semestrales o anuales llegaron a la biblioteca en un plazo no superior a tres meses después de su publicación.

Según estos criterios, una revista trimestral, por ejemplo, cumpliría la periodicidad si a lo largo del año editara 4 fascículos y el correspondiente al primer trimestre llegara a la biblioteca no después del mes de mayo; el correspondiente al segundo trimestre, no después del mes agosto; el correspondiente al tercer trimestre, no después de noviembre y el del cuarto trimestre, no después del mes de febrero del siguiente año.

Pervivencia. Se apunta como criterio de calidad la pervivencia denominada también "prestigio histórico». Se considera que el hecho de que una revista siga editándose a lo largo de varios años, sin interrupciones, es por sí mismo un elemento positivo a considerar. De hecho, todos los que trabajan con revistas conocen la dinámica de apariciones y desapariciones cada día más frecuentes. Esta es la razón por la que muchas bases de datos esperan al menos dos años antes de considerar la incorporación de una revista nueva. Cuando una revista a lo largo de su historia ha cambiado de formato e incluso de título, iniciando una nueva etapa, a efectos de contabilidad de los años de pervivencia se ha estimado el conjunto de años de las distintas etapas.

Normalización. Existe una gran cantidad de normas que afectan a la presentación de las revistas científicas y a la publicación de artículos en revistas científicas. En este estudio se han seleccionado aquéllas que parecían más relevantes, bien porque hay más 
consenso sobre la necesidad de su cumplimiento en las prácticas internacionales de publicación, bien porque están más ligadas a la difusión de las revistas. Los elementos considerados en este apartado son los siguientes:

1. UNE 50-101-90: Presentación de las publicaciones periodicas

- Apartado 4, referido al título de las publicaciones periódicas

- Apartado 5, referido a los fascículos

- Apartado 10, referido al título de cada página

2. UNE 50-133-94: Presentación de articulos en publicaciones periódicas y en serie

- Apartado 4, referido a los elementos de identificación:

- punto 2.1, relativo a la identificación de los autores

- punto 3.1, relativo a los resúmenes que deben acompañar al texto

- punto 3.2 , relativo a las palabras clave

- Anexo normativo relativo a la inclusión de instrucciones a los autores

Algunos de los apartados mencionados, especialmente el 5 y el 10 de la UNE 50101-90, se componen de diversos elementos de los cuales las revistas pueden cumplir sólo algunos, todos o ninguno. Esto obliga a tener en cuenta el cumplimiento, no del apartado completo sino de cada uno de los elementos, con el fin de matizar las situaciones intermedias que se producen con frecuencia. Especialmente complejo es el apartado que se refiere a los elementos identificativos que deben constar en la cubierta, en la cabecera del sumario y en cada una de las páginas de la publicación. En conjunto suman 19 elementos. Para aplicar un sistema evaluador no excesivamente rígido se ha adoptado el criterio de establecer unos intervalos de cumplimiento:

- si las revistas cumplen entre 0 y 7 elementos, no cumplen la norma

- si las revistas cumplen entre 8 y 13 elementos, cumplen parcialmente

- si las revistas cumplen entre 14 y 19 elementos, cumplen la norma

En el caso de las revistas que no tienen sección o serie, estos elementos son en total 18, por lo que se considera que cumplen parcialmente si tienen menos de 13 y si hacen constar 13 o más elementos, se considera que cumplen con la norma.

Consejos de Redacción. Se estudia para cada revista la existencia de un Consejo de Redacción o Consejo Editorial, así como su composición. Cuando las revistas no hacen constar su CR, se ha considerado que no lo tienen. Si los datos aportados por la revista son los nombres y apellidos de los miembros del CR, se ha solicitado a la revista por escrito la afiliación institucional de éstos. Para cada uno de los dos conjuntos de revistas, se analizan los porcentajes de revistas con y sin CR, el promedio de miembros de los CR, así como la estructura institucional de los mismos, haciendo especial mención del número de miembros de instituciones diferentes a la editora así como de los pertenecientes a instituciones extranjeras. El estudio de la pertenencia institucional de los miembros de los CR, permite detectar los niveles de endogamia de los organos de gestión de las revistas.

Sistema de evaluación de los originales. Se estudia quién interviene en el proceso de aceptación de originales, si hay un Consejo de Redacción y si hay evaluadores externos. Por otra parte, se ha diferenciado si el sistema es ciego, es decir, si los 
que juzgan el original desconocen a quién evalúan. Los datos para este apartado se han tomado directamente de la revista.

Apertura institucional. Se entiende por tal el grado de contribuciones de autores pertenecientes a instituciones diferentes de la que edita la revista, nacionales o extranjeras. La información se obtiene a partir del lugar de trabajo de los autores que lo consignan al firmar sus trabajos. Una revista puede tener poca apertura exterior (todos o la mayoría de los trabajos que publica son obra de autores de la institución que edita la revista) o, por el contrario tener una gran apertura exterior (acoge en sus páginas trabajos de autores pertenecientes a instituciones diferentes, del propio país o incluso de otros países). Se supone que el mayor grado de apertura exterior va asociado a una mayor calidad científica. El estudio del grado de apertura de cada una de las revistas cuenta con la dificultad de que los autores incumplen la norma de consignar su filiación institucional cuando firman sus trabajos y a veces cuando la aportan lo hacen de manera incompleta. Se calcula para cada revista un «índice de apertura exterior» (IAE) que se calcula dividiendo el número de instituciones diferentes (NIN) a las que pertenecen los autores entre el número total de trabajos (NT) publicados por la revista en el mismo período (1995-1996). Para este índice se han obtenido dos variantes, utilizando como numerador, en un caso, el número de instituciones nacionales diferentes $(\mathrm{IAE}=\mathrm{NIN} / \mathrm{NT}) \mathrm{y}$, en otro, el número de instituciones extranjeras diferentes (IAE=NIE/NT). Por otra parte, se ha calculado el índice de endogamia (IE=NTED/NT, donde NTDE es el número de trabajos en los que participa al menos un miembro de la institución editora, siendo NT el número de trabajos editados por la revista en el período analizado).

Difusion internacional. La difusión internacional de una revista puede medirse de muy diferentes maneras (a través de las suscripciones e intercambios, a través de los grandes centros de acceso al documento como el DSC BLL del Reino Unido, para medir su consumo a través de las peticiones de fotocopias o de duplicación de separatas de artículos, etc., a partir del estudio del factor de impacto, etc.). En este estudio se ha optado por rastrear la presencia de las revistas españolas en las bases de datos internacionales y por detectar la presencia de las revistas en Internet, sea de un modo simplemente descriptivo, sea con la posibilidad de consultar los sumarios, o finalmente, la edición electrónica de la revista. Los datos de difusión dan la medida de la visibilidad de la revista. En este sentido, puede considerarse que la «apertura exterior» da también una medida de visibilidad internacional.

Valoración de los pares. La importancia de este apartado reside en que es el único que aporta datos para evaluar directamente la calidad de las revistas. Se procedió mediante el método de encuesta dirigida individualmente a 2.800 profesores de las áreas vinculadas a las Ciencias Económicas, a partir de un listado aportado por la Dirección General de Enseñanza Superior e Investigación, que reseñaba para cada profesor su Departamento, Facultad y Universidad.

La encuesta presentaba para su valoración una relación de 48 títulos de revistas especializadas de Economía y disciplinas afines y se pedía a los especialistas que, para cada uno de los títulos de la lista, marcaran si lo conocían, si utilizaban la revista y en 
caso de conocerla, que la puntuaran entre 0 y 10 en función de su mayor o menor relevancia científica. La relación era abierta y se pedía a los encuestados que añadieran a la lista aquellos títulos que, en su opinión, deberían haber sido incluidos, aportando también para ellos su juicio y puntuación. Además de esta pregunta, la más importante, se les pedía que aportaran los títulos de aquellas revistas en las que habian publicado sus últimas contribuciones, sus preferencias en cuanto al tipo de documentos que les eran más útiles en sus trabajos y finalmente, en una pregunta abierta, se solicitaba su opinión sobre la valoración de publicaciones científicas en general, rogándoles que aportaran cuantas sugerencias estimaran oportunas.

En todo momento el equipo investigador ha sido consciente del riesgo inherente a este tipo de encuestas. Es difícil no tener presente el sesgo que puede haber en las puntuaciones, favoreciendo aquellas revistas en las que habitualmente se publica, penalizando con bajas puntuaciones a las revistas dirigidas por equipos de "escuelas" diferentes a la del especialista que cumplimenta la encuesta, etc. A pesar de todo, se consideró que merecía la pena correr el riesgo y, adoptando una serie de medidas correctoras, se envió la encuesta que ha tenido, en términos generales, una respuesta en torno al $30 \%$ y ha aportado una visión muy rica sobre las revistas españolas de los diferentes subsectores que componen el área de las Ciencias Económicas y Empresariales.

\section{Resultados de la investigación}

A continuación se aportan los datos globales más relevantes obtenidos a partir de la aplicación del método propuesto. Los resultados individualizados por revistas pueden verse en Román Román, A. y Giménez Toledo, E. (12).

\subsection{Periodicidad}

Las 48 revistas analizadas anuncian la siguiente periodicidad: revistas semanales: 1 ; revistas mensuales: 3 ; revistas bimestrales: 3 ; revistas trimestrales: 13 ; revistas cuatrimestrales: 15 ; revistas semestrales: 11 ; revistas anuales: 2 . S6́lo un $42 \%$ de las revistas cumple adecuadamente la periodicidad.

\subsection{Pervivencia}

La pervivencia media de las revistas españolas de Economía es de 21,1 años.

\subsection{Normalización}

Como se ha señalado en el apartado 3 relativo a la metodología, para evaluar la normalización de las revistas científicas se han seleccionado las normas más relevantes, en la medida en que inciden de forma más directa en la difusión internacional de las publicaciones. Las siguientes tablas muestran el porcentaje de revistas que cumplen cada una de las normas consideradas en este estudio. 
Tabla I

Revistas de Economía.

Normalizacion: elementos identificativos en cubierta

\begin{tabular}{|c|c|c|c|c|c|c|}
\hline & \multicolumn{7}{|c|}{ Cubierta } \\
\cline { 2 - 7 } Cumplen la norma & Titulo & $N .^{\circ}$ vol. & Sección & Núm. fasc. & ISSN & Fecha publ. \\
\hline $\begin{array}{c}\text { N. } .^{\circ} \text { absoluto } \\
\text { Porcentaje }\end{array}$ & 48 & 16 & 2 & 45 & 3 & 43 \\
\hline
\end{tabular}

Tabla II

Revistas de Economía.

Normalización: elementos identificativos en cabecera del sumario

\begin{tabular}{|c|c|c|c|c|c|c|}
\hline & \multicolumn{7}{|c|}{ Cubierta } \\
\cline { 2 - 7 } Cumplen la norma & Ttulo & $N^{\circ}$ vol. & Sección & Núm. fasc. & ISSN & Fecha publ. \\
\hline $\begin{array}{c}\text { N. } .^{\circ} \text { absoluto } \\
\text { Porcentaje }\end{array}$ & 23 & 9 & 0 & 17 & 0 & 15 \\
\hline
\end{tabular}

Tabla III

Revistas de Economía.

Normalización: elementos identificativos en cada página de la revista

\begin{tabular}{|c|c|c|c|c|c|c|c|}
\hline \multirow{2}{*}{$\begin{array}{c}\text { Cumplen la } \\
\text { norma }\end{array}$} & $\begin{array}{c}\text { Titulo } \\
\text { revista }\end{array}$ & Fecha & $\begin{array}{c}N^{\circ} \\
\text { vol. }\end{array}$ & $\begin{array}{c}N^{\circ} \\
\text { fasc. }\end{array}$ & $\begin{array}{c}N^{\circ} \\
\text { página }\end{array}$ & $\begin{array}{c}\text { Título } \\
\text { articulo }\end{array}$ & $\begin{array}{c}\text { Nombre } \\
\text { autor }\end{array}$ \\
\hline $\begin{array}{c}\text { N. } .^{\circ} \text { absoluto } \\
\text { Porcentaje }\end{array}$ & $\begin{array}{c}20 \\
41,7\end{array}$ & $\begin{array}{c}14 \\
29,2\end{array}$ & $\begin{array}{c}6 \\
12,5\end{array}$ & $\begin{array}{c}13 \\
27,1\end{array}$ & $\begin{array}{c}47 \\
97,9\end{array}$ & $\begin{array}{c}36 \\
75,0\end{array}$ & $\begin{array}{c}28 \\
58,3\end{array}$ \\
\hline
\end{tabular}

Tabla IV

Revistas de Economía.

Cumplimiento UNE 50-133-94

\begin{tabular}{|l|r|r|r|r|r|r|}
\hline \multicolumn{1}{|c|}{ Normas consideradas } & St & $\%$ & No & $\%$ & Parcialmente & $\%$ \\
\hline Resumen en inglés & 24 & 50 & 20 & 41,67 & 4 & 8,33 \\
Resumen en español & 25 & 52,08 & 17 & 35,42 & 6 & 12,50 \\
Palabras clave en inglés & 15 & 31,25 & 30 & 62,50 & 3 & 6,25 \\
Palabras clave en español & 15 & 31,25 & 29 & 60,42 & 4 & 8,33 \\
Afiliación institucional & 41 & 85,42 & 1 & 2,08 & 6 & 12,50 \\
Normas para los autores & 29 & 60,42 & 18 & 37,50 & 1 & 2,08 \\
Título uniforme & 45 & 93,75 & 3 & 6,25 & 0 & 0 \\
\hline
\end{tabular}




\subsection{Consejos de Redacción}

El análisis de los Consejos de Redacción pretende comprobar si éstos reúnen las características adecuadas para constituirse en mecanismos efectivos de calidad editorial de una revista. Sólo cinco de las 48 revistas evaluadas no tienen Consejo de Redacción o al menos no lo hacen constar. La tabla $\mathrm{V}$ aporta los datos considerados más relevantes para las revistas de Economía evaluadas. Por otra parte, la figura 1 refleja no sólo la proporción de revistas que cuentan con Consejos de Redacción sino también la composición de los mismos, atendiendo especialmente al nivel de endogamia y exogamia. Cabe destacar la predominante participación de miembros de instituciones españolas en los Consejos de Redacción, que para las revistas de Economía es del $97 \%$. Este dato se complementa al indicar que sólo 9 títulos de los 48 evaluados tienen participación de instituciones no españolas.

\section{Tabla V}

\section{Revistas de Economía: Consejos de Redacción}

\begin{tabular}{|l|l|}
\hline Promedio de miembros en el Consejo de Redacción & 10 \\
Número de instituciones nacionales diferentes & 98 \\
Número de instituciones extranjeras diferentes & 16 \\
\hline
\end{tabular}

\section{Figura 1}

Revistas de Economía: Perfil de los Consejos de Redacción

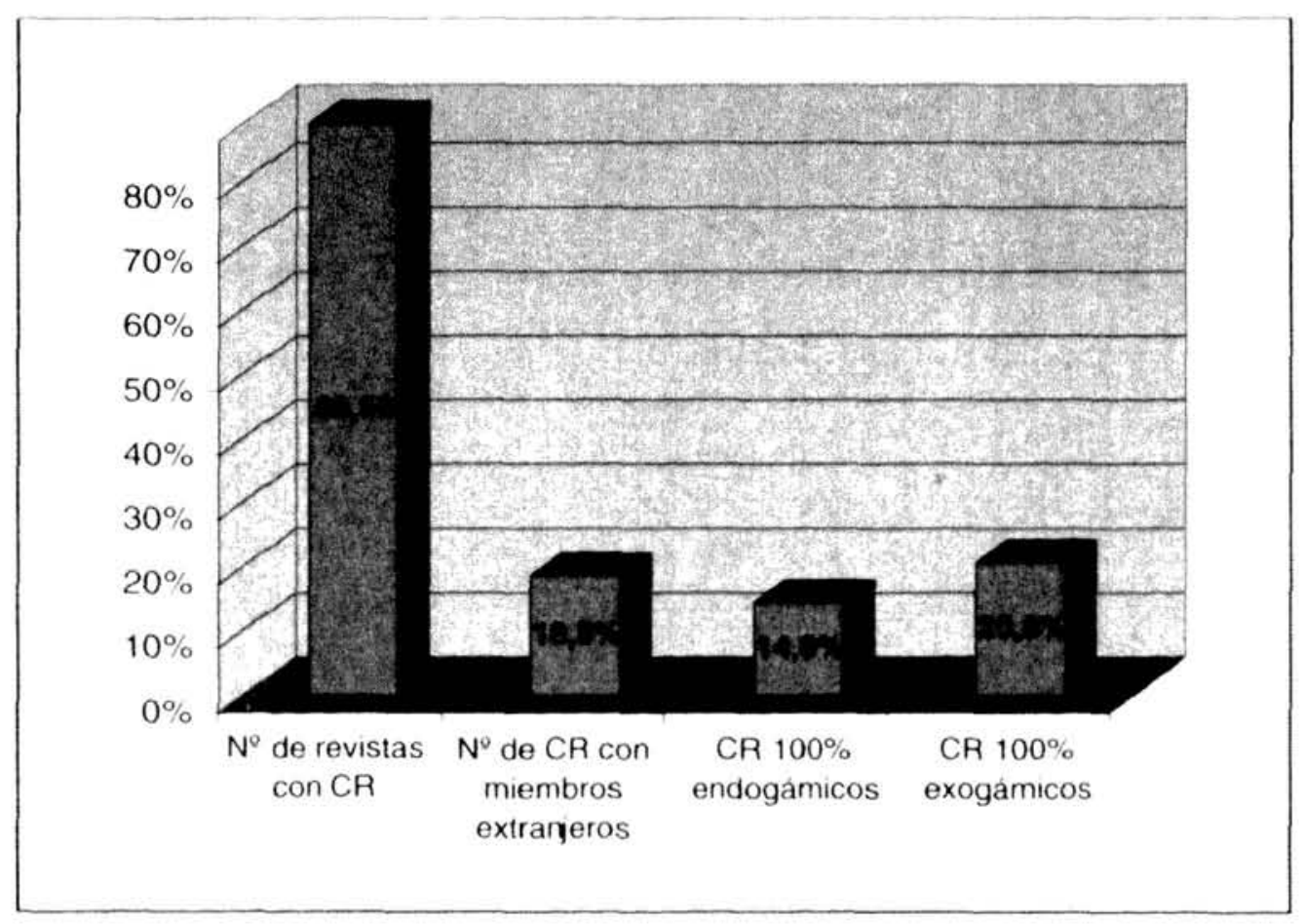




\subsection{Sistemas de evaluación de originales para su publicación}

En las revistas que no cuentan con Consejo de Redacción, la decisión sobre qué originales publicar se supone que es tomada por la Dirección de la revista. En los demás casos, interviene en la decisión el CR que en 18 revistas utiliza también evaluadores externos para el estudio de los originales. 13 de las revistas practican la evaluación anónima o sistema ciego.

\subsection{Apertura exterior}

El análisis de las instituciones de trabajo de los autores que publican en las revistas evaluadas ha permitido calcular el índice de apertura exterior, referido tanto a instituciones nacionales como extranjeras, así como el índice de endogamia.

De las 48 revistas evaluadas, 19 tienen un índice de apertura exterior nacional superior a 0,5 , mientras que al referirnos al índice de apertura exterior extranjero sólo hay dos que superen ese valor, señal de una escasa participación de instituciones extranjeras diferentes.

El índice de endogamia es bajo en términos generales: sólo 11 revistas están por encima de 0,1 .

\subsection{Difusión}

Las revistas españolas de Economía tienen una escasa visibilidad en las bases de datos internacionales. De la relación de títulos sometidos a evaluación, destaca el hecho de que hay 18 revistas que ni siquiera aparecen en Ulrich's Periodicals Directory (13), una de las bases de datos consultadas que, como es sabido, se nutre de la información enviada por los propios editores de las revistas. Otras 15 revistas sólo están recogidas en una base de datos. Dos títulos encabezan la lista de presencia en bases de datos internacionales al estar recogidos en cinco de ellas.

\section{Tabla VI}

\section{Revistas de Economía: presencia en bases de} datos internacionales

\begin{tabular}{|l|c|}
\hline \multicolumn{1}{|c|}{ Base de datos } & $\begin{array}{c}\text { Núm. de revistas } \\
\text { españolas } \\
\text { que recoge }\end{array}$ \\
\hline AGRIS & 6 \\
CAB-ABSTRACTS & 8 \\
DELPHES & 0 \\
PAIS & 4 \\
ECONLIT & 5 \\
HISTORICAL ABSTRACTS & 2 \\
IBSS & 5 \\
HAPI & 1 \\
FRANCIS & 0 \\
BLDS & 1 \\
ULRICH"S PERIODICALS DIR. & 30 \\
\hline
\end{tabular}


Por otra parte, hay 10 publicaciones que ofrecen información a distintos niveles en Internet.

\subsection{Valoración de los pares}

A partir de la tabulación de las encuestas, se han calculado las puntuaciones medias recibidas por cada revista, y se han ponderado estas puntuaciones multiplicándolas por el número de votos recibidos por cada una y dividiendo el producto por el número total de votos emitidos. Esto da un cociente, VP (valoración de los pares), que permite comparar el resultado de las puntuaciones recibidas por las revistas. A efectos de no comparar ámbitos de especialidad muy dispares, se ha procedido a agrupar las revistas valoradas en función de su contenido, siguiendo la clasificación temática asignada a las revistas en el Directorio de Revistas de Ciencias Sociales y Humanas (14). A partir de la asignación mencionada a diferentes especialidades, se han integrado en áreas similares a las reconocidas o establecidas por la Dirección General de Enseñanza Superior para los departamentos universitarios. Se ofrecen, pues, los resultados de la valoración de las revistas por los pares para los siguientes grupos de revistas:

\section{Economía aplicada}

La valoración media de los pares para esta área es de 1,47 , siendo la puntuación máxima para este grupo 4,06 y la mínima 0,34 .

\section{Economía general y Teoría económica}

En este grupo la valoración media otorgada por los pares fue 1,74 , siendo la puntuación máxima 3,60 y la mínima 0,28 .

\section{Economía agraria}

De las tres revistas que pertenecen a esta área, sólo una supera la puntuación media que es 0,73 . La puntuación máxima es 1,20 y la mínima 0,42 .

\section{Economía financiera, Contabilidad y Empresa}

La valoración media de los pares en esta área es 0,86, siendo la puntuación máxima 1,39 y la mínima 0,25 .

\section{Historia económica}

La puntuación máxima alcanzada en este grupo es de 0,67 y la mínima 0,41 , siendo la puntuación media 0,53 .

La fórmula de valoración de los pares no ha sido aplicada a aquellas revistas que han recibido menos de un $5 \%$ de los votos totales emitidos.

\section{Discusión y conclusiones}

En conjunto se ha optado por construir una metodología de evaluación que incluyera los parámetros de calidad comunes a tres de los modelos descritos al comienzo de este trabajo: prestigio histórico, difusión en B.D., apertura de las contribuciones, 
sistema de evaluación de originales y cumplimiento de la periodicidad tal y como proponen, con diferentes matices, el modelo brasileño, el colombiano y el canadiense (1) (3) (10), añadiendo aspectos como el cumplimiento de las normas internacionales de publicación de artículos científicos que propugnan los dos primeros junto con el sistema de valoración de revistas del ISI (11). Estos dos modelos, el canadiense y el empleado por el ISI, abogan además por los estudios de citas para la evaluación de la calidad de las revistas. Es evidente que con todas las limitaciones y sesgos que puedan atribuirse a estos estudios, son de gran interés. Pero no siempre es factible acometerlos, debido a los recursos disponibles. En este estudio se ha optado por someter las revistas a la evaluación de los pares.

Así pues, se ha pretendido aplicar para la evaluación de revistas científicas espanolas de Economía un modelo equilibrado que atienda a aspectos tanto formales como de contenido y que permita esbozar un mapa suficientemente detallado de la situación. Podría y quizá debería completarse con el estudio del factor de impacto de las revistas, por ejemplo, o del nivel de consumo medido a través de las estadísticas de las peticiones de fotocopias en los servicios de acceso al documento de las diferentes bibliotecas universitarias y científicas, pero ello traería consigo una complejidad aún mayor y la necesidad de ampliar considerablemente los recursos y el tiempo necesario.

En la evaluación de las revistas hay que diferenciar con claridad los objetivos perseguidos: uno es el de construir una herramienta sólida y actualizable que permita el seguimiento de las revistas de manera continuada y por lo tanto sirva, entre otros propósitos, como estímulo a los editores para mejorar sus productos, o como elemento de ayuda en los procesos evaluadores. Otro objetivo, menos práctico pero muy interesante, es el de conocer a fondo los hábitos de publicación de los científicos de un área concreta del conocimiento.

En este estudio se han mezclado un poco los dos aspectos y ello, por una parte, ha permitido obtener unos resultados muy interesantes, aún a costa de complicar los métodos y el trabajo. Es evidente que, por ejemplo, la encuesta dirigida a los especialistas, aporta información muy sustanciosa sobre la valoración que hacen de las revistas, pero tanto su elaboración y envío, como sobre todo su tratamiento posterior, suponen un trabajo considerable, complejo y con problemas metodológicos de difícil tratamiento, especialmente en materias como la Economía.

Sin embargo, es difícil obviar alguna forma de pronunciamiento de los especialistas en la evaluación de las revistas científicas. Habría que buscar un método más ágil y que pudiera dinamizar el trabajo, habida cuenta de que un elemento importante es la actualización de los datos.

Se propone pues una simplificación del modelo de valoración con parámetros de calidad objetivos, que se puedan comprobar y obtener directamente de los ejemplares de la revista y de la consulta a los diferentes recursos de información en línea y en Internet (para la difusión, especialmente) tal y como se han estudiado en el caso de las revistas de Economía, exceptuando la consulta a los pares. Esto permitiría una actualización anual de los datos, dando una mayor utilidad a la herramienta así construida.

El contraste con la opinión de los especialistas podría hacerse en periodos de tiempo mayores y ni siquiera para completar la valoración cualitativa de las revistas, sino al inicio del trabajo, para seleccionar las revistas sobre las que hacer el estudio, tal y como se hace en la Universidad de Sao Paulo (1). Es decir: se propone recurrir a los 
especialistas para que categoricen las revistas en tres grupos: muy nuclear en la disciplina, medianamente nuclear en la disciplina, marginal a la disciplina. Serviría como un filtro inicial, para limitar las revistas a evaluar en cada área.

Si en un próximo futuro se plantea la necesidad de hacer la evaluación de las revistas científicas en todas las disciplinas, es evidente que habrá que optar por modelos de evaluación ágiles y objetivos y por equipos más o menos estables que se ocupen de mantener la información sobre las revistas, garantizando la actualización de los datos.

Como última consideración en cuanto a conclusiones metodologicas se refiere, habría que señalar la importancia de integrar la evaluación de las revistas científicas españolas en algún sistema supranacional de evaluación. A este respecto, se debe subrayar la importancia que puede tener para las revistas españolas participar en el proyecto iberoamericano para crear un sistema de información pluridisciplinar, LATINDEX, que integre la producción científica del área iberoamericana.

En cuanto a los resultados obtenidos, los datos ponen de manifiesto la escasísima difusión internacional como principal problema de las revistas españolas. En una gran mayoría de los casos esta deficiencia está en relación con otras carencias detectadas: mal cumplimiento de la periodicidad, falta de normalización, niveles considerables aún de endogamia en la gestion y poca apertura institucional en las contribuciones. A pesar de ello, los datos apuntan a una mejora clara de la situación especialmente en las revistas más jóvenes.

\section{Agradecimientos}

El presente trabajo se realizó en el marco de la Acción Especial APC970038, financiada por la Dirección General de Enseñanza Superior, Programa Sectorial de Promoción General del Conocimiento. Los autores quieren agradecer la colaboración prestada en el tratamiento estadístico de los datos por Marcial Montero Ramos.

\section{Bibliografia}

1. KRZYZANOWSKI, R. F.; GONZAGA FERREIRA, M. C. Evaluación de publicaciones cientificas y técnicas brasileñas. En CETTO, A. M., y Alonso, O. (comp.), Revistas científicas en América Latina. México: ICSU, etc., 1999, p. 406-423.

2. MORICONI VALERIO, P. Espelho da Ciência: avaliaçao do Programa Setorial de Publicaçoes em Ciencia e Tecnologia da FINEP. Rio de Janeiro: FINEP, IBICT, 1994.

3. JACK GÓMEZ, Y. A propósito de un ejercicio de evaluación de seriadas científicas. En: CETTO, A. M.; ALONSO, O. (comp.), Revistas científicas en América Latina. México: ICSU, etc., 1999, p. 375-389.

4. ORTEGA, C.; PLAZA, L. M.; MARTIN, M. J.; URDIN, M. C. Spanish scientific and technical journals. State of the art. Scientometrics. 1992, vol. 24, n. ${ }^{\circ} 1$, p. 21-42

5. ORTEGA FERNANDEZ, C.; PLAZA GOMEZ, L. M. Las revistas españolas de ciencia y tecnología como vehículos de difusión de la investigación cientifica. Revista Española de Documentación Cientifica. 1993, vol. 16, n. ${ }^{\circ} 3$, p. 221-227

6. DELGADO LOPEZ-COZAR, E. Evaluación y aplicación de las normas de presentación de publicaciones periódicas: revisión bibliográfica. Revista Española de Documentación Cientifica. 1997, vol. 20, n. ${ }^{\circ} 1$, p. 39-51. 
7. SALES HEREDIA, P.; GARCIA MARIN, A.; ROMAN ROMAN, A. Las revistas cientrficas, transmisoras del conocimiento. Una metodologfa de evaluación. En Organización del conocimiento en sistemas de información y documentación. Zaragoza: Universidad de Zaragoza-ISKO, 1997, p.271-283

8. GARCIA MARIN, A.; ROMAN ROMAN, A. Las publicaciones periódicas de Historia Antigua, Prehistoria y Arqueologia: difusión internacional. Trabajos de Prehistoria. 1998, vol. 55, n. $^{\circ} 1$, p. $139-146$.

9. JONES, M. J.; BRINN, T.; PENDLEBURY, M. Journal evaluation methodologies: a balanced response. Omega: International Journal of Management Science. 1996, vol. 24, n. ${ }^{0} 5$, p. 607-612.

10. NATIONAL RESEARCH COUNCIL OF CANADA. Indicators of quality for research journals: a report of the advisory board on scientific publications to the research community based on a report of a task force on quality assessment. 1981. Informe.

11. TESTA, J. The ISI Database: the journal selection process. The ISI Essays. http://www:isinet.com/whatshot/essays/essay9701.html.

12. ROMAN ROMAN, A.; GIMENEZ TOLEDO, E. Las revistas españolas de Economía: una propuesta de evaluación científica. Información Comercial Española. Revista de Economia. 1999 (en evaluación)

13. Ulrich's periodicals directory. Münich: Bowker-Saur, 1998. Edición en CD-ROM

14. VILLAGRA RUBIO, A. Directorio de revistas españolas en Ciencias Sociales y Humanas. Madrid: CINDOC, 1998, p.105 y ss. 